\title{
Mammographic density and breast cancer in women from high risk families
}

Teresa Ramón y Cajal ${ }^{1}$, Isabel Chirivella ${ }^{2}$, Josefa Miranda ${ }^{3}$, Alexandre Teule ${ }^{4}$, Ángel Izquierdo ${ }^{5}$, Judith Balmaña ${ }^{6}$, Ana Beatriz Sánchez-Heras ${ }^{7}$, Gemma Llort ${ }^{8}$, David Fisas', Virginia Lope ${ }^{9,10,11}$, Elena Hernández-Agudo ${ }^{12}$, María José Juan-Fita ${ }^{13}$, Isabel Tena ${ }^{14}$, Luis Robles ${ }^{15}$, Carmen Guillén-Ponce ${ }^{16}$, Pedro Pérez-Segura ${ }^{17}$, Mari Sol Luque-Molina ${ }^{18}$, Susana Hernando-Polo ${ }^{19}$, Mónica Salinas ${ }^{4}$, Joan Brunet ${ }^{5,20}$, María Dolores Salas-Trejo ${ }^{3}$, Agustí Barnadas ${ }^{1}$ and Marina Pollán ${ }^{9,10,11^{*}}$

\begin{abstract}
Introduction: Mammographic density (MD) is one of the strongest determinants of sporadic breast cancer (BC). In this study, we compared MD in BRCA1/2 mutation carriers and non-carriers from BRCA1/2 mutation-positive families and investigated the association between $M D$ and $B C$ among BRCA1/2 mutation carriers per type of mutation and tumor subtype.

Methods: The study was carried out in 1039 female members of BRCA1 and BRCA2 mutation-positive families followed at 16 Spanish Genetic Counseling Units. Participants' density was scored retrospectively from available mammograms by a single blinded radiologist using a 5-category scale (<10 \%, 10-25 \%, 25-50 \%, 50-75 \%, >75 \%). In $\mathrm{BC}$ cases, we selected mammograms taken prior to diagnosis or from the contralateral breast, whereas, in non-cases, the last screening mammogram was evaluated. MD distribution in carriers and non-carriers was compared using ordinal logistic models, and the association between MD and BC in BRCA1/2 mutation carriers was studied using logistic regression. Huber-White robust estimators of variance were used to take into account correlations between family members. A similar multinomial model was used to explore this association by BC subtype.

Results: We identified and scored mammograms from 341 BRCA1, 350 BRCA2 mutation carriers and 229 non-carriers. Compared to non-carriers, MD was significantly lower among BRCA2 mutation carriers (odds ratio $(\mathrm{OR})=0.71$; $P$-value $=0.04)$, but not among $B R C A 1$ carriers $(\mathrm{OR}=0.84 ; P$-value $=0.33)$. MD was associated with subsequent development $\mathrm{BC}(\mathrm{OR}$ per category of $\mathrm{MD}=1.45 ; 95 \%$ confidence interval $=1.18-1.78, P$-value $<0.001)$, with no significant differences between $B R C A 1$ and BRCA2 mutation carriers ( $P$-value $=0.48$ ). Finally, no statistically significant differences were observed in the association of MD with specific BC subtypes.
\end{abstract}

Conclusions: Our study, the largest to date on this issue, confirms that MD is an independent risk factor for all BC subtypes in either BRCA1 and BRCA2 mutation carriers, and should be considered a phenotype risk marker in this context.

\section{Introduction}

Mammographic density (MD) is one of the strongest determinants of breast cancer (BC) in most ethnic groups. In general population studies, higher $\mathrm{MD}$ is consistently associated with a higher BC risk [1]. Individual variation of MD does not only depend on well-established

\footnotetext{
* Correspondence: mpollan@isciii.es

${ }^{9}$ National Center for Epidemiology, Carlos III Institute of Health, Monforte de Lemos 5, 28029 Madrid, Spain

${ }^{10}$ Consortium for Biomedical Research in Epidemiology and Public Health (CIBERESP), Carlos III Institute of Health, Madrid, Spain

Full list of author information is available at the end of the article
}

epidemiologic factors, such as age, parity, menopausal status, external hormonal manipulation or body mass index (BMI), but based on family studies [2-7], it also depends on the as yet unknown genetic background.

Knowledge of the genetic basis of MD could be a major milestone in $\mathrm{BC}$ risk prediction and prevention. Previous candidate gene analysis and linkage studies have shown either inconclusive or discordant results to date. In contrast, genome-wide association studies have started to identify some genetic variants that could explain part of the variation in MD [8]. Furthermore, 
some of the well-established BC susceptibility variants are also associated with $\mathrm{MD}$ variability $[9,10]$.

Germline mutations in BRCA1 and BRCA2 genes are the most frequent cause of strong genetic predisposition to breast and ovarian cancer. In the last decades, several studies have evaluated whether the mutational status of both genes is associated with differences in MD compared to the general population. Therefore, whereas earlier studies with limited numbers of patients [11-16] had conflicting results, two larger and well-conducted studies compared $\mathrm{MD}$ in either affected or healthy BRCA1/2 mutation carriers [17] and in mutation carriers versus women with low to average risk [18]. In one of them, authors proved $\mathrm{MD}$ to be an independent risk factor for $\mathrm{BC}$ in carriers [17]. In contrast, findings from a recent study did not reveal any association between baseline MD and subsequent risk of $\mathrm{BC}$ among carriers [19].

In this paper, we aimed to compare MD in Spanish BRCA1/2 mutation carriers and non-carriers and assess the potential impact of MD on subsequent $\mathrm{BC}$ risk among carriers according to the mutated gene and pathologic subtype.

\section{Methods}

A total of 1,039 women were enrolled from 509 breast and ovarian cancer families who had been counseled and tested for mutations in BRCA1 and BRCA2 genes, from January 1993 to December 2012, and followed up at 16 Cancer Genetic Units in Spain. The study was approved by national and regional Ethics Committees (see Acknowledgments for details) and conducted in compliance with the Helsinki Declaration. All women were older than 18 years and gave written informed consent for the study.

Eligible participants were women from mutation-positive families, including $B R C A 1 / 2$ mutation carriers and true mutation-negative women. Women diagnosed with $\mathrm{BC}$ were considered cases, whereas those individuals with no personal history of $\mathrm{BC}$ were regarded as non-cases or controls.

Structured questionnaires collecting baseline information were administered by investigators during either the highrisk breast screening or the post-test visit. Apart from demographic characteristics, data for other covariates were collected, such as the genetic condition, age, weight and height, reproductive history, menopausal status, the use of exogenous hormonal supplements and history of riskreducing surgery up to the time of the study mammogram. Controls' weight and height were measured by the investigator at the enrollment visit. Tumor subtype, weight and height at the time of the scored mammogram were extracted from the medical charts of $\mathrm{BC}$ patients.

Mammograms were either obtained from the radiological chart or provided by the participants. Due to the retrospective design of our study, we considered density measures from analog and digital mammographic films. For cancer patients, we aimed to collect the earliest mammogram obtained before diagnosis (within a 10-year period and identical menopausal status) and excluded the films from the affected breast. When unavailable, we used the mammogram of the unaffected contralateral breast taken at the time of cancer diagnosis. To make the age of controls at the time of the mammogram more comparable with those of women with $\mathrm{BC}$, we attempted to obtain the latest available mammogram. For each participant, films from craniocaudal and mediolateral oblique views were requested. There were 119 women who either did not have a suitable mammogram (110) or the quality of the film was insufficient (9), rendering a total of 920 individuals for whom the mammogram was available and MD reading was feasible. There were 691 women carrying a deleterious mutation in the $B R C A 1$ or $B R C A 2$ (341 women with $B R C A 1$ and 350 with $B R C A 2$ ) genes and 229 women were relatives without a mutation.

Breast density was visually assessed by one experienced radiologist, with high intra-observer concordance [20]. The radiologist was blinded to the diagnosis, the carrier status and the referral centre of the mammogram. MD was classified using the Boyd semiquantitative scale into 6 categories, namely $0 \%,<10 \%, 10-25 \%, 25-50 \%, 50-75 \%$ and $>75 \%$. The radiologist provided a single reading per participant, considering the craniocaudal and mediolateral oblique views in both breasts when available. For those breast cancer cases without previous mammography, the two views of the contralateral breast were read.

The association between MD and the carrier status as well as other variables of interest was assessed in healthy women using an ordinal logistic regression model. This procedure results from fitting k-1 logistic regression models, dichotomizing MD into two groups, i.e., high versus low MD, using all possible cutoffs. This model, also known as the proportional odds model, assumes that odds ratios (ORs) remain constant irrespective of the cutoff chosen, but allows the intercepts to differ. The following variables were included as possible confounders: age at mammogram, BMI, menopausal status (premenopause/natural menopause/surgical menopause), parity (nulliparous/parous), type of image (analog/digital) and carrier status (non-carrier, BRCA1, BRCA2). The correlation between members of the same family was taken into account using Huber-White robust estimators of variance, considering individuals clustered inside families [21]. The Brant test was used to verify the proportional odds assumption.

The association between $\mathrm{MD}$ and subsequent $\mathrm{BC}$ development was evaluated using a logistic regression model, adjusted for age at mammogram, menopausal status (premenopause/natural menopause/surgical menopause), BMI, parity, age at first live birth, use of hormonal 
replacement therapy (HRT) (never/ever), type of image and time elapsed from mammogram to either $\mathrm{BC}$ diagnosis (cases) or end of follow up (non-cases). The consistency of the association between $\mathrm{MD}$ and $\mathrm{BC}$ was explored performing subgroup analysis in models including $\mathrm{MD}$ as a continuous variable and an interaction term between MD and each of the explanatory variables already mentioned. Finally, possible differences in the effect of MD per BC subtype were assessed using a multinomial logistic model, adjusted for the same confounders and considering the following subtypes: 1) hormonal receptor positive (estrogen receptor (ER) or progesterone receptor (PR)) and human epidermal growth factor receptor 2 (HER2) negative, 2) HER2-positive, 3) triple-negative tumors, and 4) unknown subtype. Again, Huber-White robust estimators of variance were used in all instances to take into account the correlation between relatives [21]. Sensitivity analyses were carried out considering only those cases with mammograms taken 6 months or more before $\mathrm{BC}$ diagnosis. All statistical analyses were performed by using the STATA version 12.0 software program (Stata Corp, College Station, TX, USA).

\section{Results}

\section{Baseline characteristics}

Baseline characteristics of our population according to $B R C A 1 / 2$ mutation carrier status and development of breast cancer are shown in Table 1. Carriers were younger at the scored mammogram (mean age was 41 years, regardless of the mutated gene and disease condition) compared with non-carriers (45 years; $P$ value $<0.001$ ). In addition, carriers were more likely to have ever used hormone preventive treatment $(1.4 \%$ vs $0.4 \% ; P$ value $=0.06)$ and have undergone surgical menopause at the time of the selected mammogram $(10.3 \%$ vs $1.7 \%$; $P$ value $<0.001)$. There were 31 women, 21 BRCA1 and 10 BRCA2 mutation carriers, with a previous diagnosis of ovarian cancer, and four of them (2 BRCA1 and 2 BRCA2 mutation carriers) subsequently developed a breast tumor.

Among 691 BRCA1/2 mutation carriers, 233 women developed invasive breast cancer (111 and 122 with $B R C A 1$ and BRCA2 mutations, respectively), and 15 patients had ductal carcinoma in situ (3 BRCA1 and 12 BRCA2 mutation carriers, respectively), whereas 7 women had invasive breast cancer and the other 3 women developed ductal carcinoma in situ, among 229 non-carriers. Regarding pathologic subtypes, 54 cases, 2 of them among non-carriers, could not be classified due to lack of information on hormone receptor expression (11 cases) or HER2 status (54 cases). As expected, BRCA2 mutation carriers were more likely to have hormone receptor positive tumors and no expression of HER2 (71 \% versus $26 \%$; $P$ value $<0.001)$, whereas $B R C A 1$ mutation carriers had a higher proportion of triple-negative tumors (66\% versus $14 \%$; $P$ value $<0.001$ ).

When we looked specifically at BRCA1/2 mutation carriers, breast cancer patients had a lower BMI compared to controls, with $92 \%$ of cases versus $85 \%$ of controls having $\mathrm{BMI}<30(P$ value $=0.03)($ Table 1$)$. No differences were seen in the age at the time of scored mammogram, but analog mammography was more frequent among cases $(84 \%$ versus $30 \%)$. Finally, MD was higher in affected carriers, with $33 \%$ of them in the two highest categories (MD $>50 \%)$ as opposed to $22 \%$ of controls $(P$ value $=0.001)$. Menopausal status, parity, age at first birth, oral contraceptives and hormonal replacement therapy were not significantly different between both groups. As expected, surgical menopause was more frequent among controls $(14 \%$ versus $4 \%$; $P$ value $<0.001)$.

\section{Association of mammographic density with other variables}

The association of all categories of mammographic density and explanatory variables is shown in Table 2 . Given the small number of women with $0 \% \mathrm{MD}$, the two first categories were combined in subsequent analyses. In all instances, ORs were adjusted for age at mammogram, BMI, menopausal status (premenopause/natural menopause/surgical menopause), type of mammogram, parity, carrier status and subsequent development of BC. We did not find a significant association between carrying a $B R C A 1 / 2$ mutation and a lower $\mathrm{MD}(\mathrm{OR}=0.77 ; 95 \%$ $\mathrm{CI}=0.57,1.05)$. However, when $B R C A 1$ and $B R C A 2$ mutation carriers were considered separately, $B R C A 2$ mutation carriers had a statistically significant lower MD $(\mathrm{OR}=0.71 ; 95 \% \mathrm{CI}=0.51,0.98: P$ value $=0.04)$, but no differences were observed for BRCA1 mutation carriers $(P$ value $=0.332)$. Most risk factors considered, including age at mammography, BMI, menopausal status and parity were, as expected, significantly associated with MD. Moreover, MD was higher among women who subsequently developed a breast tumor $(\mathrm{OR}=1.69 ; 95 \% \mathrm{CI}=1.24$, $2.31 ; P$ value $=0.001)$, Finally, neither age at first birth nor hormonal interventions such as HRT or oral contraceptives $(\mathrm{OC})$ significantly modified MD in our study.

\section{Effect of mammographic density in development of breast cancer in BRCA1/2 mutation carriers}

The distribution of MD in $\mathrm{BC}$ cases and non-cases for women who were non-carriers and BRCA1 and BRCA2 mutation-carriers is presented in Fig. 1. Cases tended to be more frequently classified in the higher $\mathrm{MD}$ categories. Given the limited number of $\mathrm{BC}$ cases among the noncarriers group, we did not evaluate the potential different effect of MD on cancer risk in non-carriers. Table 3 shows the association between $\mathrm{MD}$ and subsequent development of $\mathrm{BC}$ among $B R C A 1 / 2$ mutation carriers. After adjusting 
Table 1 Characteristics of the women from BRCA1/2 families included in the study

\begin{tabular}{|c|c|c|c|c|c|c|}
\hline \multirow{3}{*}{ Variable } & \multicolumn{3}{|c|}{ BRCA1/2 mutation carriers } & \multicolumn{3}{|c|}{ Relatives without mutation } \\
\hline & \multirow{2}{*}{$\begin{array}{l}\text { Without BC } \\
N=443\end{array}$} & \multirow{2}{*}{$\begin{array}{l}\text { BC patients } \\
N=248\end{array}$} & \multirow[t]{2}{*}{$P$ value $^{a}$} & \multirow{2}{*}{$\begin{array}{l}\text { Without BC } \\
N=219\end{array}$} & \multirow{2}{*}{$\begin{array}{l}\text { BC patients } \\
N=10\end{array}$} & \multirow[t]{2}{*}{$P$ value $^{\mathrm{a}}$} \\
\hline & & & & & & \\
\hline \multicolumn{3}{|l|}{ Affected gene } & 0.183 & & & . \\
\hline$B R C A 1$ & $227(51.2 \%)$ & $114(46.0 \%)$ & & & & \\
\hline$B R C A 2$ & $216(48.8 \%)$ & $134(54.0 \%)$ & & & & \\
\hline \multicolumn{2}{|l|}{ Age at mammography } & & 0.909 & & & 0.907 \\
\hline Mean (SD) & $41.6(11.5)$ & $41.5(10.2)$ & & $45.6(11.8)$ & $45.2(11.2)$ & \\
\hline \multicolumn{2}{|l|}{ Body mass index } & & 0.030 & & & 0.948 \\
\hline$<18.5$ & $6(1.4 \%)$ & $7(2.8 \%)$ & & $11(5.0 \%)$ & $0(0.0 \%)$ & \\
\hline $18.5-24.9$ & $259(58.5 \%)$ & $151(60.9 \%)$ & & $120(54.8 \%)$ & $7(70.0 \%)$ & \\
\hline $25-29.9$ & $110(24.8 \%)$ & $69(27.8 \%)$ & & $56(25.6 \%)$ & $2(20.0 \%)$ & \\
\hline $30-34.9$ & 49 (11.1\%) & $19(7.7 \%)$ & & $28(12.8 \%)$ & $1(10.0 \%)$ & \\
\hline$>=35$ & $19(4.3 \%)$ & $2(0.8 \%)$ & & $4(1.8 \%)$ & $0(0.0 \%)$ & \\
\hline Unknown & $0(0.0 \%)$ & $0(0.0 \%)$ & & $11(5.0 \%)$ & $0(0.0 \%)$ & \\
\hline \multicolumn{2}{|l|}{ Menopausal status ${ }^{b}$} & & 0.239 & & & 0.576 \\
\hline Premenopausal & $310(70.0 \%)$ & $184(74.2 \%)$ & & $147(67.1 \%)$ & 7 (70.0 \%) & \\
\hline Postmenopausal & $133(30.0 \%)$ & $64(25.8 \%)$ & & 72 (32.9 \%) & $3(30.0 \%)$ & \\
\hline \multicolumn{2}{|l|}{ Type of menopause $\mathrm{e}^{\mathrm{b}, \mathrm{c}}$} & & $<0.001$ & & & 0.154 \\
\hline Natural & $73(16.5 \%)$ & $53(21.4 \%)$ & & $69(95.8 \%)$ & $2(66.7 \%)$ & \\
\hline Surgery & $60(13.5 \%)$ & $11(4.4 \%)$ & & $3(4.2 \%)$ & 1 (33.3 \%) & \\
\hline \multicolumn{2}{|l|}{ Time since menopause $\mathrm{e}^{\mathrm{b}, \mathrm{c}}$} & & 0.100 & & & 1.000 \\
\hline$<=5$ years & $31(7.0 \%)$ & $19(7.7 \%)$ & & $27(12.3 \%)$ & $1(10.0 \%)$ & \\
\hline $6-10$ years & $34(7.7 \%)$ & $8(3.2 \%)$ & & 17 (7.8 \%) & $1(10.0 \%)$ & \\
\hline$>10$ years & $63(14.2 \%)$ & 35 (14.1\%) & & $28(12.8 \%)$ & $1(10.0 \%)$ & \\
\hline Unknown & $5(1.1 \%)$ & 2 (0.8 \%) & & $0(0.0 \%)$ & $0(0.0 \%)$ & \\
\hline \multicolumn{2}{|l|}{ Nulliparous } & & 0.151 & & & 0.264 \\
\hline No & $302(68.2 \%)$ & $182(73.4 \%)$ & & $167(76.3 \%)$ & $6(60.0 \%)$ & \\
\hline Yes & $141(31.8 \%)$ & 66 (26.6\%) & & $52(23.7 \%)$ & $4(40.0 \%)$ & \\
\hline \multicolumn{2}{|l|}{ Age at first birth, years ${ }^{d}$} & & 0.110 & & & 0.464 \\
\hline$>30$ & 75 (16.9\%) & $34(13.7 \%)$ & & $46(27.5 \%)$ & $0(0.0 \%)$ & \\
\hline $26-30$ & $111(25.1 \%)$ & $64(25.8 \%)$ & & 60 (35.9\%) & $3(50.0 \%)$ & \\
\hline $21-25$ & 98 (22.1\%) & 77 (31.0\%) & & 53 (31.7\%) & $3(50.0 \%)$ & \\
\hline$<=20$ & $18(4.1 \%)$ & $7(2.8 \%)$ & & $8(4.8 \%)$ & $0(0.0 \%)$ & \\
\hline \multicolumn{2}{|l|}{ Oral contraceptives } & & 0.487 & & & 0.508 \\
\hline No & $156(35.2 \%)$ & 81 (32.7 \%) & & $58(26.5 \%)$ & $4(40.0 \%)$ & \\
\hline Yes & $239(54.0 \%)$ & $140(56.5 \%)$ & & $130(59.4 \%)$ & $6(60.0 \%)$ & \\
\hline Unknown & $48(10.8 \%)$ & 27 (10.9\%) & & $31(14.2 \%)$ & $0(0.0 \%)$ & \\
\hline \multicolumn{2}{|c|}{ Use of hormone replacement therapy ${ }^{\mathrm{b}}$} & & 0.613 & & & 0.246 \\
\hline No & $431(97.3 \%)$ & $238(96.0 \%)$ & & $205(93.6 \%)$ & $9(90.0 \%)$ & \\
\hline Current use & $3(0.7 \%)$ & $3(1.2 \%)$ & & $5(2.3 \%)$ & $1(10.0 \%)$ & \\
\hline Past use & $9(2.0 \%)$ & $7(2.8 \%)$ & & $9(4.1 \%)$ & $0(0.0 \%)$ & \\
\hline \multicolumn{3}{|c|}{ Use of hormonal preventive treatment ${ }^{\mathrm{b}}$} & 0.349 & & & \\
\hline No & $438(98.9 \%)$ & $243(98.0 \%)$ & & $219(99.5 \%)$ & $10(100 \%)$ & \\
\hline Current use & $5(1.1 \%)$ & $5(2.0 \%)$ & & $1(0.5 \%)$ & $0(0.0 \%)$ & \\
\hline
\end{tabular}


Table 1 Characteristics of the women from BRCA1/2 families included in the study (Continued)

\begin{tabular}{|c|c|c|c|c|c|c|}
\hline Type of mammogram & & & $<0.001$ & & & 0.020 \\
\hline Analog & $132(29.8 \%)$ & 208 (83.9 \%) & & $62(28.3 \%)$ & $6(60.0 \%)$ & \\
\hline Digital & $311(70.2 \%)$ & 40 (16.1\%) & & $157(71.7 \%)$ & $4(40.0 \%)$ & \\
\hline Number of projections & & & $<0.001$ & & & 0.069 \\
\hline One & $9(2.0 \%)$ & $6(2.4 \%)$ & & & & \\
\hline Two & $34(7.7 \%)$ & 74 (29.8 \%) & & $11(4.7 \%)$ & $3(25.0 \%)$ & \\
\hline Three & $2(0.5 \%)$ & $3(1.2 \%)$ & & $1(0.4 \%)$ & $0(0.0 \%)$ & \\
\hline Four & $398(89.8 \%)$ & 165 (66.5 \%) & & $223(94.9 \%)$ & $9(75.0 \%)$ & \\
\hline Mammographic density & & & 0.001 & & & 0.316 \\
\hline $0 \%$ & $6(1.4 \%)$ & $0(0.0 \%)$ & & $3(1.4 \%)$ & $1(10.0 \%)$ & \\
\hline$<=10 \%$ & 99 (22.3\%) & 35 (14.1\%) & & 44 (20.1\%) & $1(10.0 \%)$ & \\
\hline $11-25 \%$ & 89 (20.1\%) & 47 (19.0\%) & & 44 (20.1\%) & $2(20.0 \%)$ & \\
\hline $26-50 \%$ & $151(34.1 \%)$ & 85 (34.3\%) & & 72 (32.9 \%) & $3(30.0 \%)$ & \\
\hline $51-75 \%$ & $72(16.3 \%)$ & 47 (19.0\%) & & 37 (16.9\%) & $3(30.0 \%)$ & \\
\hline$>75 \%$ & $26(5.9 \%)$ & 34 (13.7 \%) & & $19(8.7 \%)$ & 0 (0.0\%) & \\
\hline Time of follow upe & & & $<0.001$ & & & 0.924 \\
\hline$<=1$ year & $207(46.7 \%)$ & $162(65.3 \%)$ & & $103(47.0 \%)$ & $4(40.0 \%)$ & \\
\hline $1-2$ years & $82(18.5 \%)$ & 30 (12.1\%) & & 39 (17.8 \%) & $2(20.0 \%)$ & \\
\hline $2-5$ years & $94(21.2 \%)$ & $30(12.1 \%)$ & & $48(21.9 \%)$ & $3(30.0 \%)$ & \\
\hline $5-10$ years & $46(10.4 \%)$ & $24(9.7 \%)$ & & $22(10.0 \%)$ & $1(10.0 \%)$ & \\
\hline$>10$ years & $14(3.2 \%)$ & $2(0.8 \%)$ & & $7(3.2 \%)$ & $0(0.0 \%)$ & \\
\hline Prophylatic mastectomy & & & 0.003 & & & \\
\hline No & $428(96.6 \%)$ & 248 (100.0 \%) & & & & \\
\hline Yes & $15(3.4 \%)$ & $0(0.0 \%)$ & & & & \\
\hline \multicolumn{7}{|l|}{ Breast cancer features } \\
\hline Histology & & & & & & . \\
\hline Ductal in situ & & $15(6.0 \%)$ & & & $3(30.0 \%)$ & \\
\hline Ductal & & 187 (75.4 \%) & & & $6(60.0 \%)$ & \\
\hline Lobular & & $12(4.8 \%)$ & & & $1(10.0 \%)$ & \\
\hline Medular & & $14(5.6 \%)$ & & & $0(0.0 \%)$ & \\
\hline Others & & $18(7.3 \%)$ & & & $0(0.0 \%)$ & \\
\hline Unknown & & $2(0.8 \%)$ & & & $0(0.0 \%)$ & \\
\hline \multicolumn{7}{|l|}{ Type of breast cancer } \\
\hline ER/PR+\&HER2- & & 99 (39.9 \%) & & & $6(60.0 \%)$ & \\
\hline HER2+ & & $23(9.3 \%)$ & & & $1(10.0 \%)$ & \\
\hline Triple-negative & & 74 (29.8 \%) & & & $1(10.0 \%)$ & \\
\hline Unknown & & $52(21.0 \%)$ & & & $2(20.0 \%)$ & \\
\hline
\end{tabular}

${ }^{a}$ Comparison between women who did and did not develop breast cancer. ${ }^{b}$ At time of mammography. ${ }^{c}$ Only postmenopausal women at time of mammography. ${ }^{\mathrm{d} O n l y}$ parous women. ${ }^{\mathrm{T}}$ Time elapsed from mammographic exploration to breast cancer diagnosis (cases) or last contact (controls). $B C$ breast cancer, $E R$ estrogen receptor, $P R$ progesterone receptor, HER2 human epidermal growth factor receptor 2

for age at mammogram, BMI, menopause (premenopause/ natural menopause/surgical menopause), parity, age at first birth, HRT, type of mammogram and time of follow up, there was a clear association between $\mathrm{MD}$ and subsequent $\mathrm{BC}$ development among BRCA1/2 mutation carriers, with a statistically significant dose-response trend $(P$ value
$<0.001)$. The OR in women with density $50-75 \%$ relative to women with density $\leq 10 \%$ was $3.24(95 \% \mathrm{CI}=1.43$, 7.35; $P$ value $=0.005)$, while women in the highest $\mathrm{MD}$ category, namely $>75 \%$, had an OR of 4.34 ( $95 \% \mathrm{CI}=1.71$, 11.1; $P$ value $=0.002$ ). The association between $\mathrm{MD}$ and $\mathrm{BC}$ tended to be stronger among $B R C A 2$ mutation carriers 
Table 2 Association of mammographic density with carrier status and other characteristics in healthy female mutation carriers (controls) from BRCA1/BRCA2 families

\begin{tabular}{|c|c|c|c|c|c|c|c|c|c|}
\hline \multirow[b]{2}{*}{ Variable } & \multirow[b]{2}{*}{ Number } & \multicolumn{5}{|c|}{ Categories of mammographic density } & \multirow[b]{2}{*}{ Odds ratio $^{a}$} & \multirow[b]{2}{*}{$95 \% \mathrm{Cl}^{\mathrm{a}}$} & \multirow[b]{2}{*}{$P$ valu } \\
\hline & & $<=10 \%$ & $11-25 \%$ & $26-50 \%$ & $51-75 \%$ & $>75 \%$ & & & \\
\hline \multicolumn{10}{|l|}{ Carrier status } \\
\hline Non-carrier & 219 & $21 \%$ & $20 \%$ & $33 \%$ & $17 \%$ & $9 \%$ & 1.00 & & \\
\hline$B R C A 1$ or $B R C A 2$ & 443 & $24 \%$ & $20 \%$ & $34 \%$ & $16 \%$ & $6 \%$ & 0.75 & $0.54,1.04$ & 0.085 \\
\hline$B R C A 1$ & 227 & $22 \%$ & $25 \%$ & $29 \%$ & $19 \%$ & $6 \%$ & 0.84 & $0.58,1.23$ & 0.376 \\
\hline$B R C A 2$ & 216 & $26 \%$ & $15 \%$ & $39 \%$ & $14 \%$ & $6 \%$ & 0.67 & $0.47,0.96$ & 0.028 \\
\hline
\end{tabular}

Age at mammography, years

\begin{tabular}{|c|c|c|c|c|c|c|c|c|c|}
\hline$<35$ & 188 & $10 \%$ & $14 \%$ & $36 \%$ & $27 \%$ & $13 \%$ & 1.00 & & \\
\hline $35-44$ & 199 & $17 \%$ & $19 \%$ & $39 \%$ & $17 \%$ & $8 \%$ & 0.94 & $0.54,1.62$ & 0.826 \\
\hline $45-54$ & 170 & $29 \%$ & $25 \%$ & $31 \%$ & $12 \%$ & $3 \%$ & 0.79 & $0.33,1.90$ & 0.603 \\
\hline$>=55$ & 105 & $49 \%$ & $25 \%$ & $24 \%$ & $3 \%$ & $0 \%$ & 0.57 & $0.15,2.20$ & 0.415 \\
\hline Linear $t$ & & & & & & & 0.97 & $0.95,0.99$ & $<0.001$ \\
\hline
\end{tabular}

Body mass index

\begin{tabular}{|c|c|c|c|c|c|c|c|c|c|}
\hline$<25$ & 396 & $10 \%$ & $17 \%$ & $39 \%$ & $24 \%$ & $10 \%$ & 1.00 & & \\
\hline $25.0-29.9$ & 166 & $36 \%$ & $26 \%$ & $28 \%$ & $7 \%$ & $3 \%$ & 0.28 & $0.19,0.40$ & $<0.001$ \\
\hline$>=30$ & 100 & $51 \%$ & $24 \%$ & $23 \%$ & $2 \%$ & $0 \%$ & 0.16 & $0.10,0.25$ & $<0.001$ \\
\hline Linear tren & & & & & & & 0.83 & $0.80,0.86$ & $<0.001$ \\
\hline
\end{tabular}

Menopausal status \& type of menopause

\begin{tabular}{|c|c|c|c|c|c|c|c|c|c|}
\hline Premenopausal & 457 & $15 \%$ & $17 \%$ & $37 \%$ & $21 \%$ & $9 \%$ & 1.00 & & \\
\hline Natural menopause & 142 & $41 \%$ & $24 \%$ & $28 \%$ & $6 \%$ & $1 \%$ & 0.72 & $0.45,1.16$ & 0.179 \\
\hline Surgical menopause & 63 & $38 \%$ & $35 \%$ & $21 \%$ & $6 \%$ & $0 \%$ & 0.56 & $0.33,0.96$ & 0.034 \\
\hline \multicolumn{10}{|l|}{ Julliparous } \\
\hline No & 460 & $29 \%$ & $23 \%$ & $33 \%$ & $13 \%$ & $2 \%$ & 1.00 & & \\
\hline Yes & 202 & $9 \%$ & $14 \%$ & $35 \%$ & $24 \%$ & $17 \%$ & 1.87 & $1.28,2.73$ & 0.001 \\
\hline
\end{tabular}

Age at first birth, years (only parous women)

\begin{tabular}{|c|c|c|c|c|c|c|c|c|c|}
\hline$>30$ & 131 & $25 \%$ & $17 \%$ & $35 \%$ & $17 \%$ & $6 \%$ & 1.00 & & \\
\hline $25-29$ & 170 & $25 \%$ & $25 \%$ & $34 \%$ & $15 \%$ & $1 \%$ & 1.17 & $0.72,1.88$ & 0.526 \\
\hline $20-24$ & 151 & $35 \%$ & $24 \%$ & $30 \%$ & $9 \%$ & $2 \%$ & 1.10 & $0.67,1.82$ & 0.706 \\
\hline$<20$ & 26 & $38 \%$ & $23 \%$ & $27 \%$ & $12 \%$ & $0 \%$ & 0.72 & $0.31,1.66$ & 0.436 \\
\hline Linear tr & & & & & & & 1.00 & $0.97,1.05$ & 0.745 \\
\hline Hormonal & & & & & & & & & \\
\hline No & 636 & $23 \%$ & $20 \%$ & $34 \%$ & $17 \%$ & $7 \%$ & 1.00 & & \\
\hline Yes & 26 & $23 \%$ & $27 \%$ & $27 \%$ & $15 \%$ & $8 \%$ & 1.71 & $0.87,3.38$ & 0.120 \\
\hline Hormonal & & & & & & & & & \\
\hline No & 656 & $23 \%$ & $20 \%$ & $34 \%$ & $17 \%$ & $7 \%$ & 1.00 & & \\
\hline Yes & 6 & $33 \%$ & $17 \%$ & $50 \%$ & $0 \%$ & $0 \%$ & 0.67 & $0.25,1.82$ & 0.434 \\
\hline Oral contro & & & & & & & & & \\
\hline No & 214 & $22 \%$ & $18 \%$ & $34 \%$ & $19 \%$ & $7 \%$ & 1.00 & & \\
\hline Yes & 369 & $22 \%$ & $21 \%$ & $33 \%$ & $17 \%$ & $7 \%$ & 0.93 & $0.67,1.27$ & 0.637 \\
\hline ype of im & & & & & & & & & \\
\hline Analog & 194 & $26 \%$ & $21 \%$ & $34 \%$ & $13 \%$ & $6 \%$ & 1.00 & & \\
\hline Digital & 468 & $22 \%$ & $20 \%$ & $34 \%$ & $18 \%$ & $7 \%$ & 1.09 & $0.79,1.50$ & 0.588 \\
\hline
\end{tabular}


Table 2 Association of mammographic density with carrier status and other characteristics in healthy female mutation carriers (controls) from BRCA1/BRCA2 families (Continued)

\begin{tabular}{lllllllll}
\hline Number of projections & & & & & & & & \\
One or two & 53 & $15 \%$ & $17 \%$ & $40 \%$ & $21 \%$ & $8 \%$ & 1.00 & \\
Three or Four & 609 & $24 \%$ & $20 \%$ & $33 \%$ & $16 \%$ & $7 \%$ & 1.17 & $0.70,1.94$ \\
\hline
\end{tabular}

${ }^{a}$ Adjusted for age at mammogram, body mass index, menopause (premenopausal, natural menopause, surgical menopause), type of mammogram, parity (nulliparous versus parous ), carrier status (non-carrier, BRCA1, BRCA2) and subsequent development of breast cancer (no, yes)

(OR per increase in one category of MD of 1.60 in $B R C A 2$ and of 1.37 in BRCA1 carriers), but these differences were not statistically significant $(P$ value for heterogeneity $=0.449)$. Sensitivity analysis including only the cases with a mammogram obtained at least 6 months before cancer diagnosis had comparable results (see the central columns in Table 3). Finally, given that most mammograms from $\mathrm{BC}$ cases were analog, the last columns present the association between $\mathrm{MD}$ and $\mathrm{BC}$ using only analog images (Table 3 ). ORs tended to be stronger in this case, particularly for BRCA2 mutation carriers. The analysis of digital images was hampered by the small number of $\mathrm{BC}$ cases available ( 40 cases: $6 \mathrm{MD}<=10 \%$, $6 \mathrm{MD}=11-25 \%, 17 \mathrm{MD}=26-50 \%, 9 \mathrm{MD}=51-75 \%$ and $2 \mathrm{MD}>75 \%$ ) and no association between $\mathrm{MD}$ and $\mathrm{BC}$ was observed (ORs of 1.23, 1.94, 1.88 and 1.22, respectively; none of the ORs were statistically significant).

The association between MD (as an ordinal variable) and $\mathrm{BC}$ per category of the other explanatory variables among mutation carriers is presented in Fig. 2. The OR represents the risk excess associated with an increase in one category of MD. In general, a consistent effect of $\mathrm{MD}$ on $\mathrm{BC}$ risk was seen within all subgroup analyses. Even though the association seemed to be less marked among women older than 45 years at the time of mammography, those who were nulliparous, and those who had not taken $\mathrm{OC}$, none of these differences were statistically significant. The smaller number of women who reported the use of HRT was too small to reach conclusions in this group. The association with the type of image was stronger for analog mammograms, but most cases had analog mammograms, whereas the opposite was true for non-cases. Finally, MD increased the risk of all pathologic subtypes BC subtypes in a similar way ( $P$ value for heterogeneity $=0.638$ ).

\section{Discussion}

In this case-control study of women from high-risk breast cancer families, we confirmed that breast density was an independent risk factor for $\mathrm{BC}$ among BRCA1 and BRCA2 mutation carriers. Compared to women with breast density $<10 \%$, those with densities $>50 \%$ had at

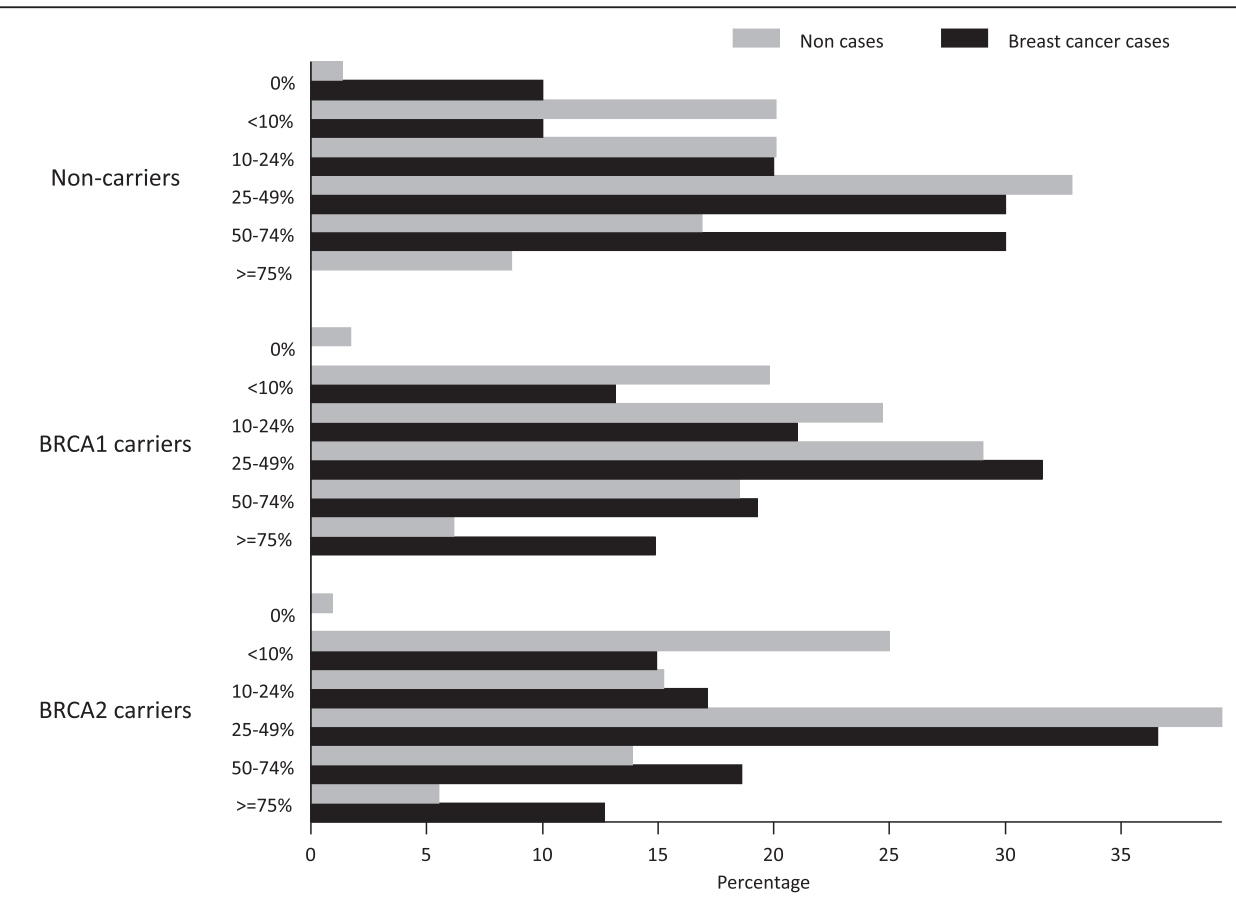

Fig. 1 Distribution of mammographic density in breast cancer cases and non-cases in the following groups: non-carriers, BRCA1 mutation carriers and BRCA2 mutation carriers 
Table 3 Association of mammographic density with subsequent breast cancer in BRCA1/2 mutation carriers

\begin{tabular}{|c|c|c|c|c|c|c|c|c|c|c|c|c|c|c|}
\hline \multirow[b]{2}{*}{$\begin{array}{l}\text { Mammographic } \\
\text { density }\end{array}$} & \multirow[b]{2}{*}{ Non-cases } & \multicolumn{4}{|c|}{ All cases included } & \multicolumn{4}{|c|}{$\begin{array}{l}\text { Excluding cases without a } \\
\text { mammogram taken at least } \\
6 \text { months before diagnosis }\end{array}$} & \multicolumn{5}{|c|}{ Including only analog images } \\
\hline & & $\begin{array}{l}\text { BC } \\
\text { cases }\end{array}$ & $O R^{a}$ & $95 \% \mathrm{Cl}^{\mathrm{a}}$ & $P$ value $^{a}$ & $\begin{array}{l}\mathrm{BC} \\
\text { cases }^{\mathrm{a}}\end{array}$ & $\mathrm{OR}^{\mathrm{a}}$ & $95 \% \mathrm{Cl}^{\mathrm{a}}$ & $P$ value $^{\mathrm{a}}$ & Non-cases & $\begin{array}{l}\mathrm{BC} \\
\text { cases }\end{array}$ & $O R^{b}$ & $95 \% \mathrm{Cl}^{\mathrm{b}}$ & $P$ value $^{\mathrm{b}}$ \\
\hline \multicolumn{15}{|l|}{$B R C A 1+B R C A 2$} \\
\hline$<=10 \%$ & 105 & 35 & 1.00 & & & 12 & 1.00 & & & 36 & 29 & 1.00 & & \\
\hline $1125 \%$ & 89 & 47 & 1.51 & $0.74,3.06$ & 0.255 & 18 & 1.76 & $0.70,4.41$ & 0.228 & 29 & 41 & 1.74 & $0.72,4.19$ & 0.216 \\
\hline $26-50 \%$ & 151 & 85 & 1.85 & $0.90,3.82$ & 0.095 & 39 & 2.26 & $0.94,5.44$ & 0.070 & 45 & 68 & 1.77 & $0.75,4.15$ & 0.191 \\
\hline $51-75 \%$ & 72 & 47 & 3.24 & $1.43,7.35$ & 0.005 & 21 & 3.67 & $1.37,9.80$ & 0.010 & 15 & 38 & 4.88 & $1.72,13.9$ & 0.003 \\
\hline$>75 \%$ & 26 & 34 & 4.34 & $1.71,11.1$ & 0.002 & 17 & 8.94 & $2.86,28.0$ & $<0.001$ & 7 & 32 & 9.45 & $2.73,32.7$ & $<0.001$ \\
\hline Linear trend & 443 & 248 & 1.45 & $1.18,1.78$ & $<0.001$ & 107 & 1.64 & $1.28,2.09$ & $<0.001$ & 132 & 208 & 1.68 & $1.31,2.15$ & $<0.001$ \\
\hline \multicolumn{15}{|l|}{$B R C A 1$} \\
\hline$<=10 \%$ & 49 & 15 & 1.00 & & & 3 & 1.00 & & & 15 & 14 & 1.00 & & \\
\hline $11-25 \%$ & 56 & 24 & 2.18 & $0.85,5.58$ & 0.103 & 8 & 2.71 & $0.63,11.7$ & 0.183 & 15 & 20 & 1.78 & $0.50,6.48$ & 0.370 \\
\hline $26-50 \%$ & 66 & 36 & 1.90 & $0.78,4.67$ & 0.160 & 14 & 3.06 & $0.73,12.9$ & 0.127 & 23 & 30 & 1.15 & $0.39,3.39$ & 0.798 \\
\hline $51-75 \%$ & 42 & 22 & 2.53 & $0.89,7.18$ & 0.082 & 10 & 4.22 & $0.93,19.2$ & 0.063 & 9 & 21 & 3.68 & $0.75,18.1$ & 0.109 \\
\hline$>75 \%$ & 14 & 17 & 5.42 & $1.79,16.4$ & 0.003 & 9 & 12.0 & $2.20,65.1$ & 0.004 & 3 & 16 & 7.49 & $1.57,35.7$ & 0.012 \\
\hline Linear trend & 227 & 114 & 1.37 & $1.06,1.76$ & 0.015 & 44 & 1.65 & $1.17,2.32$ & 0.004 & 65 & 101 & 1.51 & $1.08,2.12$ & 0.017 \\
\hline \multicolumn{15}{|l|}{$B R C A 2$} \\
\hline$<=10 \%$ & 56 & 20 & 1.00 & & & 9 & 1.00 & & & 21 & 15 & 1.00 & & \\
\hline $11-25 \%$ & 33 & 23 & 1.29 & $0.45,3.68$ & 0.639 & 10 & 1.70 & $0.47,6.19$ & 0.419 & 14 & 21 & 2.21 & $0.76,6.46$ & 0.146 \\
\hline $26-50 \%$ & 85 & 49 & 2.03 & $0.65,6.34$ & 0.222 & 25 & 3.36 & $0.89,12.7$ & 0.074 & 22 & 38 & 3.85 & $1.06,13.9$ & 0.040 \\
\hline $51-75 \%$ & 30 & 25 & 5.37 & $1.35,21.4$ & 0.017 & 11 & 7.38 & $1.26,43.1$ & 0.027 & 6 & 17 & 11.2 & $1.82,68.9$ & 0.009 \\
\hline$>75 \%$ & 12 & 17 & 4.82 & $1.10,21.1$ & 0.037 & 8 & 13.4 & $2.33,77.3$ & 0.004 & 4 & 16 & 18.8 & $3.06,114.9$ & 0.002 \\
\hline Linear trend & 216 & 134 & 1.60 & $1.13,2.28$ & 0.008 & 63 & 1.95 & $1.30,2.91$ & 0.001 & 67 & 107 & 2.11 & $1.40,3.16$ & $<0.001$ \\
\hline
\end{tabular}

${ }^{a}$ Odds ratio $(O R), 95 \% \mathrm{Cl}$ and $P$ values adjusted for age at mammogram, menopausal status premenopausal, natural menopause, surgical menopause), body mass index, parity (nulliparous, parous), age at first live birth, use of hormonal replacement treatment (yes, no), type of image (analog, digital) and time elapsed from mammogram to breast cancer diagnosis (cases) or end of follow up (non-cases). ${ }^{b} \mathrm{OR}, 95 \% \mathrm{Cl}$ and $P$ values adjusted for the above-mentioned variables except type of image

least threefold increased risk of developing a breast tumor. The association between MD and the risk of subsequent $\mathrm{BC}$ seemed to be stronger for $B R C A 2$ than for BRCA1 mutation carriers, but the sample size was insufficient to detect statistical differences between them. Our results also confirmed an association between MD and all pathologic BC subgroups. Finally, we found that mutation carriers had lower MD estimations compared to noncarriers and this difference remained significant among $B R C A 2$ mutation carriers.

The role of MD as a risk predictor for $\mathrm{BC}$ has relevant implications in screening and preventive interventions, particularly in highly motivated women with a family history of the disease. Our results were consistent with early evidence for the association between $\mathrm{BC}$ risk and $\mathrm{MD}$ among women from high-risk families $[6,7]$ and later findings on BRCA1 and BRCA2 mutation carriers [17, 18], assessed by computerized methods for obtaining quantitative measures of MD. Initial case-control studies evaluating the association between family history, MD and subsequent risk of $\mathrm{BC}$ were completed by Martin et al. [7] in a sample of 2,322 subjects from three Canadian screening programs. In that population, $\mathrm{MD}$ explained $14 \%$ of the association between family history and $\mathrm{BC}$ risk, being considered an intermediate marker for $\mathrm{BC}$. Moreover, women with breast density $>50 \%$ had threefold higher risk of $\mathrm{BC}$ than those with $<10 \%$ density $(95 \% \mathrm{CI}=2.17,4.15)$. Our OR for carriers with $>50 \%$ breast density are in agreement with those previously reported in our country among BC screening attendants, using the same semiquantitative visual scale [22]. In that study, the risk excess associated with higher MD was similar in women with and without a family history of BC, similar to the findings of the Nurses' Health Study [23].

Raising the question of the potential impact of MD on women with a germline mutation in BRCA1 and BRCA2 genes, Mitchell et al. evaluated the association between $\mathrm{MD}$ and $\mathrm{BC}$ risk among 342 women from the EMBRACE study, 206 of whom were $B R C A$ mutation carriers [17]. In spite of some methodological differences and a limited 


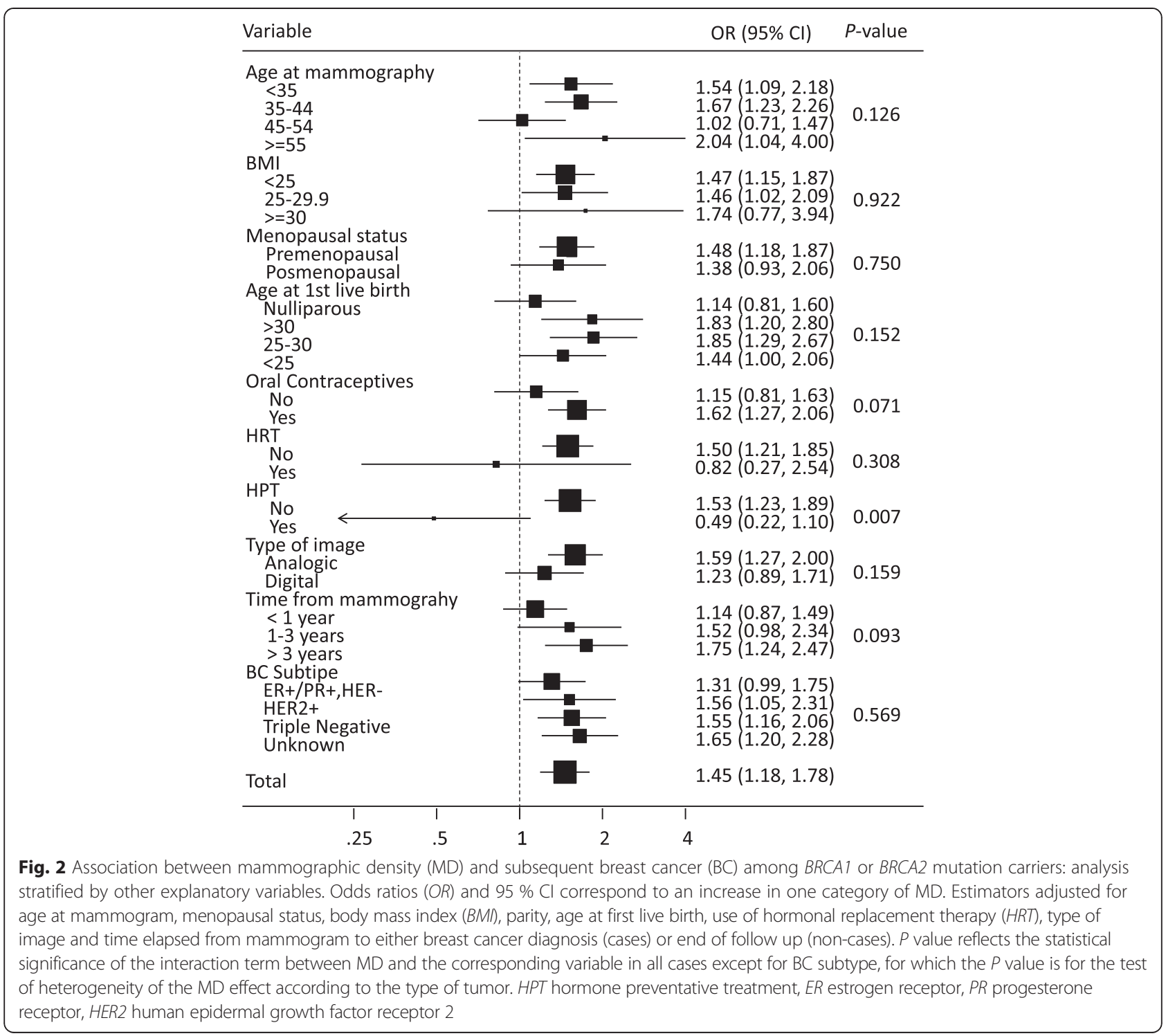

sample of participants, their results were comparable to ours. Assessing MD with a semi-automatic tool, the authors estimated the OR for $\mathrm{BC}$ among carriers with $\geq 50 \%$ breast density to be 2.27 (95\% CI $=0.70,7.39$ ) compared with carriers who had $<10 \%$ breast density, while using a qualitative scale (Wolfe's), the highest density group had a risk excess close to $3(\mathrm{OR}=2.78)$ [17]. In contrast, a recent prospective nested case-control study investigating the same issue in a cohort of 462 BRCA mutation carriers enrolled in a BC screening program failed to confirm this association [19]. These negative results could be partially explained by facts such as the relatively small sample size and small number of cases, the high proportion of patients with a prior diagnosis and treatment of BC (19\%), the older mean age at the baseline mammogram, and the lack of information on BMI, one of the most important established confounding factors for
MD. This confounding effect is particularly relevant when MD is assessed using computer-assisted tools, given the strong correlation between BMI and the non-dense area $[24,25]$. Due to the potential impact of cancer treatment on $\mathrm{MD}$, and similar to the EMBRACE study, we restricted our study to include only women with first diagnosis of $\mathrm{BC}$ as cases. Indeed, Passaperuma et al. did not provide information relative to adjuvant cancer therapy, which could have acted as a confounding factor [19]. In fact, based on the effect of tamoxifen in reducing MD, research has been lately focused on evaluating the potential use of MD variation as a target for identifying women who would benefit from this drug either as preventive or adjuvant therapy [26-28].

Despite initial conflicting results from a small series of patients, two later studies investigating the effect of mutation status on MD patterns have reported similar results. 
The first study was based on 505 retrospectively obtained mammographic readings from 342 women and found no significant difference in MD between carriers and noncarriers, either as a group or when mutated genes were considered separately [17]. In fact, the mean density was slightly lower in carriers than non-carriers. A second study compared MD between 143 healthy carriers and 119 women determined to be at low to average risk of $\mathrm{BC}$ [18]. In agreement with our results, the authors also found a marginally lower, albeit not significant, MD among carriers after adjustment for age and BMI. In our study, based on a larger sample size, mutation carriers had lower breast density compared to non-carriers, although this effect was only statistically significant for $B R C A 2$ mutation carriers.

As regards $\mathrm{BC}$ subtypes, previous studies have addressed the association between $M D$ and $B C$ in the general population with inconsistent results [22, 29-32]. Therefore, whereas a few studies have suggested that MD is mainly associated with hormone receptor positive tumors [32], others have reported a similar [22, 30] or event stronger association with triple-negative tumors, especially for women aged $<55$ years [29]. In addition, and similar to our results in BRCA1/2 mutation carriers, a recent study in screening participants in Spain proved that high MD was associated with all histologic subtypes of $\mathrm{BC}$ [22].

Our study had several limitations, mostly related to its retrospective nature. Indeed, the heterogeneity of mammograms due to the use of multiple mammographers, the inclusion of both digital and analog images and differences in methodology, such as the subjective visual assessment of breast density and the use of semiquantitative assessments, limit the comparability of our results with other studies. Regarding the use of digital and analog mammograms, density tends to be lower when estimated via digital mammography and most images for $\mathrm{BC}$ cases were analog (84\% analog versus $16 \%$ digital). Owing to this, we were able to show a clear effect of $\mathrm{MD}$ on $\mathrm{BC}$ risk in general and also restricting the analysis to cases and controls with analog images, but no effect was observed using digital mammograms, probably due to the small number of BCs in this category and the fact that only eight of them were classified in the extreme categories. Future studies are needed to confirm these results in mutation carriers studied with digital mammograms. Last, although the plan in original design of our study was to use healthy true negative BRCA1/2 mutation carriers and phenocopies as controls for comparing the effect of MD on cancer risk between carriers and non-carriers, the smaller number of cases in this group did not allow us to accomplish this goal.

The strengths of our study include the fact that this is the largest cohort of women with a BRCA1/2 mutation and $\mathrm{MD}$ data ever published. In spite of being a multicentric study, a single blinded radiologist, who is a highly reputed expert on mammographic reading, with a very high internal consistency [20], assessed all mammograms using a semiquantitative scale that has proved to be associated with subsequent development of BC [22]. Finally, restricting the analysis to $\mathrm{BC}$ patients with mammograms obtained 6 months before the diagnosis and the adjustment for all known confounders of MD provides compelling support for our results.

\section{Conclusions}

This is the largest study to date to assess the influence of $\mathrm{MD}$ on $\mathrm{BC}$ risk and is concordant with previously published results in that $\mathrm{MD}$ is an independent risk factor among BRCA1/2 mutation carriers. Even though MD was not increased among mutation carriers compared to non-carriers, high MD increased the risk of all pathologic subtypes of $\mathrm{BC}$. Prospective studies are needed to clarify the impact of density variations on $\mathrm{BC}$ risk and provide a more pro-active prevention strategy for women harboring $B R C A 1 / 2$ mutations.

\section{Abbreviations}

BC: breast cancer; BMI: body mass index; ER: estrogen receptor; HER2: human epidermal growth factor receptor 2; HPT: hormone preventative treatment; HRT: hormonal replacement treatment; MD: mammographic density; OC: oral contraceptives; OR: odds ratio; PR: progesterone receptor.

\section{Competing interests}

The authors declare that they have no competing interests.

\section{Authors' contributions}

MP, TRyC, ICh and DST conceived the study and discussed their idea with the other authors, and all of them contributed to the design of the research proposals that provided financial support. JM, VL and DF helped design the questionnaire and were in charge of organizing mammographic readings. TRyC, ICh, AT, Al, JB, ABSH, GLI, EHA, MJJF, IT, LR, CGP, PPS, MSLM, SHP, MS, $J B$ and $A B$ recruited women from $B R C A 1 / 2$ families and collected the mammograms and information needed together with the pathologic records of BC cases. MP performed the statistical analysis and results were circulated and commented on by all the authors. MP, TRyC and ICh drafted the first version that was critically reviewed by the other authors, who made suggestions on how to improve it. All of them have read and approved the final version of the manuscript.

\section{Acknowledgements}

This work was supported by three research grants, PS09/01006, PS09/01024 and PS09/01721, from Spain's Health Research Fund (Fondo de Investigación Sanitaria); and a grant from the Spanish Federation of Breast Cancer Patients (Federación Española de Cáncer de Mama) (FECMA 485 EPY 1170-10). The authors also acknowledge the contribution of the Asociación Española contra el Cáncer (AECC), Carlos III Health Institute; RTICC; and the Catalan Health Institute and Autonomous Government of Catalonia (grant numbers: RD06/0020/1051, RD06/0020/1050, RD12/0036/0008, RD12/0036/0031,

PI10/01422, PI13/00285, PIE13/00022 and SGR290). This project was approved by the following ethical boards: Comité de Ética de la Investigación y de Bienestar Animal CElyBA, Instituto de Salud Carlos III; Comité de Ética de la Investigación Clínica Hospital de la Santa Creu y Sant Pau, Barcelona; Comité de Ética de la Investigación Clínica Hospital Universitari de Girona Doctor Josep Trueta, Girona; Comité de Ética de la Fundación INCLIVA, Hospital Clínico Universitario, Valencia; Comité de Ética de la Investigación Clínica del Hospital Universitario 12 de Octubre, Madrid, and Comité de Ética de la Investigación Clínica del Hospital Ramón y Cajal, Madrid. 


\section{Author details}

${ }^{1}$ Medical Oncology Department, Hospital Santa Creu I Sant Pau, Barcelona, Spain. ${ }^{2}$ Medical Oncology Department, Hospital Clinico Universitario de Valencia, Valencia, Spain. ${ }^{3}$ Foundation General Directorate Public Health and Foundation for the Promotion of Health and Biomedical Research in the Valencian Region, FISABIO - Public Health, Valencia, Spain. ${ }^{4}$ Hereditary Cancer Program, Catalan Institue of Oncology-IDIBELL, Barcelona, Spain. ${ }^{5}$ Hereditary Cancer Program, Catalan Institute of Oncology-IDIBGI, Girona, Spain. ${ }^{6}$ Medical Oncology Deartment, Hospital Vall Hebron/Vall Hebron Institute of Oncology, Barcelona, Spain. ${ }^{7}$ Medical Oncology Department, Hospital Universitario de Elche, Elche, Spain. ${ }^{8}$ Genetic Counseling Unit, Corporació Sanitaria Parc tauli, Consorci Sanitari de Terrassa, Terrasa, Spain. ${ }^{9}$ National Center for Epidemiology, Carlos III Institute of Health, Monforte de Lemos 5, 28029 Madrid, Spain. ${ }^{10}$ Consortium for Biomedical Research in Epidemiology and Public Health (CIBERESP), Carlos III Institute of Health Madrid, Spain. ${ }^{11}$ Consortium Cancer Epidemiology Research Group, Oncology and Hematology Area, IIS Puerta de Hierro (IDIPHIM), Madrid, Spain. ${ }^{12}$ Breast Cancer Unit, Clinical Research Programme, Spanish National Cancer Center (CNIO), Madrid, Spain. ${ }^{13}$ Medical Oncology Department, Foundation of the Valencian Oncologic Institute, Valencia, Spain. ${ }^{14}$ Medical Oncology Department, Hospital Provincial de Castellón, Castellón, Spain. ${ }^{15}$ Medical Oncology Department, Hospital 12 de Octubre, Madrid, Spain. ${ }^{16}$ Medical Oncology Department, Hospital Universitario Ramón y Cajal, IRYCIS, Madrid, Spain. ${ }^{17}$ Medical Oncology Department, Hospital Clínico San Carlos, Madrid, Spain. ${ }^{18}$ Gynecological Department, Hospital Gregorio Marañón, Madrid, Spain. ${ }^{19}$ Medical Department, Hospital Fundación Alcorcón, Alcorcón, Spain. ${ }^{20}$ Medical Sciences Department, School of Medicine, University of Girona, Girona, Spain.

\section{Received: 15 December 2014 Accepted: 24 June 2015}

\section{Published online: 11 July 2015}

\section{References}

1. McCormack VA, dos Santos Silva I. Breast density and parenchymal patterns as markers of breast cancer risk: a meta-analysis. Cancer Epidemiol Biomarkers Prev. 2006;15:1159-69.

2. Kataoka M, Antoniou A, Warren R, Leyland J, Brown J, Audley T, et al. Genetic models for the familial aggregation of mammographic breast density. Cancer Epidemiol Biomarkers Prev. 2009;18:1277-84.

3. Haars G, van Noord PA, van Gils CH, Peeters PH, Grobbee DE. Heritable aspects of dysplastic breast glandular tissue (DY). Breast Cancer Res Treat. 2004:87:149-56.

4. Kaprio J, Alanko A, Kivisaari L, Standertskjold-Nordenstam CG. Mammographic patterns in twin pairs discordant for breast cancer. Br J Radiol. 1987;60:459-62

5. Boyd NF, Dite GS, Stone J, Gunasekara A, English DR, McCredie MR, et al. Heritability of mammographic density, a risk factor for breast cancer. N Engl J Med. 2002;347:886-94.

6. Boyd NF, Lockwood GA, Martin LJ, Knight JA, Jong RA, Fishell E, et al. Mammographic densities and risk of breast cancer among subjects with a family history of this disease. J Natl Cancer Inst. 1999;91:1404-8.

7. Martin LJ, Melnichouk O, Guo H, Chiarelli AM, Hislop TG, Yaffe MJ, et al. Family history, mammographic density, and risk of breast cancer. Cancer Epidemiol Biomarkers Prev. 2010;19:456-63.

8. Lindstrom S, Vachon CM, Li J, Varghese J, Thompson D, Warren R, et al. Common variants in ZNF365 are associated with both mammographic density and breast cancer risk. Nat Genet. 2011;43:185-7.

9. Vachon CM, Scott CG, Fasching PA, Hall P, Tamimi RM, Li J, et al. Common breast cancer susceptibility variants in LSP1 and RAD51L1 are associated with mammographic density measures that predict breast cancer risk. Cancer Epidemiol Biomarkers Prev. 2012;21:1156-66.

10. Fernandez-Navarro P, Pita G, Santamarina C, Moreno MP, Vidal C, Miranda-Garcia J, et al. Association analysis between breast cancer genetic variants and mammographic density in a large population-based study (Determinants of Density in Mammographies in Spain) identifies susceptibility loci in TOX3 gene. Eur J Cancer. 2013;49:474-81.

11. Chang J, Yang WT, Choo HF. Mammography in Asian patients with BRCA1 mutations. Lancet. 1999;353:2070-1.

12. Tilanus-Linthorst M, Verhoog L, Obdeijn IM, Bartels K, Menke-Pluymers M, Eggermont $A$, et al. A BRCA1/2 mutation, high breast density and prominent pushing margins of a tumor independently contribute to a frequent false-negative mammography. Int J Cancer. 2002;102:91-5.

13. Hamilton LJ, Evans AJ, Wilson AR, Scott N, Cornford EJ, Pinder SE, et al Breast imaging findings in women with BRCA1- and BRCA2-associtaed breast carcinoma. Clin Radiol. 2004;59:895-902

14. Kaas R, Kroger R, Peterse $J$, Hart AA, Muller SH. The correlation of mammographic-and histologic patterns of breast cancers in BRCA1 gene mutation carriers, compared to age-matched sporadic controls. Eur Radiol. 2006;16:2842-8.

15. Huo Z, Giger ML, Olopade Ol, Wolverton DE, Weber BL, Metz CE, et al. Computerized analysis of digitized mammograms of BRCA1 and BRCA2 gene mutation carriers. Radiology. 2002;225:519-26.

16. Li H, Giger ML, Huo Z, Olopade OI, Lan L, Weber BL, et al. Computerized analysis of mammographic parenchymal patterns for assessing breast cancer risk: effect of ROI size and location. Med Phys. 2004;31:549-55.

17. Mitchell G, Antoniou AC, Warren R, Peock S, Brown J, Davies R, et al. Mammographic density and breast cancer risk in BRCA1 and BRCA2 mutation carriers. Cancer Res. 2006;66:1866-72.

18. Gierach GL, Loud JT, Chow CK, Prindiville SA, Eng-Wong J, Soballe PW, et al. Mammographic density does not differ between unaffected BRCA1/2 mutation carriers and women at low-to-average risk of breast cancer. Breast Cancer Res Treat. 2010;123:245-55.

19. Passaperuma K, Warner E, Hill KA, Gunasekara A, Yaffe MJ. Is mammographic breast density a breast cancer risk factor in women with BRCA mutations? J Clin Oncol. 2010;28:3779-83.

20. Garrido-Estepa M, Ruiz-Perales F, Miranda J, Ascunce N, Gonzalez-Roman I, Sanchez-Contador C, et al. Evaluation of mammographic density patterns: reproducibility and concordance among scales. BMC Cancer. 2010;10:485.

21. Hardin JW, Hilbe JM. Generalized Linear Models and Extensions. College Station: Stata Press; 2007

22. Pollan M, Ascunce N, Ederra M, Murillo A, Erdozain N, Ales-Martinez J, et al. Mammographic density and risk of breast cancer according to tumor characteristics and mode of detection: a Spanish population-based case-control study. Breast Cancer Res. 2013;15:R9.

23. Yaghjyan L, Colditz GA, Rosner B, Tamimi RM. Mammographic breast density and breast cancer risk by menopausal status, postmenopausal hormone use and a family history of breast cancer. Cancer Causes Control. 2012;23:785-90.

24. Assi V, Warwick J, Cuzick J, Duffy SW. Clinical and epidemiological issues in mammographic density. Nat Rev Clin Oncol. 2012;9:33-40.

25. Nguyen TL, Schmidt DF, Makalic E, Dite GS, Stone J, Apicella C, et al. Explaining variance in the cumulus mammographic measures that predict breast cancer risk: a twins and sisters study. Cancer Epidemiol Biomarkers Prev. 2013;22:2395-403.

26. Cuzick J, Warwick J, Pinney E, Duffy SW, Cawthorn S, Howell A, et al. Tamoxifen-induced reduction in mammographic density and breast cancer risk reduction: a nested case-control study. J Natl Cancer Inst. 2011;103:744-52.

27. Ko KL, Shin IS, You JY, Jung SY, Ro J, Lee ES. Adjuvant tamoxifen-induced mammographic breast density reduction as a predictor for recurrence in estrogen receptor-positive premenopausal breast cancer patients. Breast Cancer Res Treat. 2013;142:559-67.

28. Kim J, Han W, Moon HG, Ahn S, Shin HC, You JM, et al. Breast density change as a predictive surrogate for response to adjuvant endocrine therapy in hormone receptor positive breast cancer. Breast Cancer Res. 2012;14:R102.

29. Bertrand KA, Tamimi RM, Scott CG, Jensen MR, Pankratz V, Visscher D, et al. Mammographic density and risk of breast cancer by age and tumor characteristics. Breast Cancer Res. 2013:15:R104.

30. Phipps Al, Buist DS, Malone KE, Barlow WE, Porter PL, Kerlikowske K, et al. Breast density, body mass index, and risk of tumor marker-defined subtypes of breast cancer. Ann Epidemiol. 2012;22:340-8.

31. Yaghjyan L, Colditz GA, Collins LC, Schnitt SJ, Rosner B, Vachon C, et al. Mammographic breast density and subsequent risk of breast cancer in postmenopausal women according to tumor characteristics. J Natl Cancer Inst. 2011;103:1179-89.

32. Conroy SM, Pagano I, Kolonel LN, Maskarinec G. Mammographic density and hormone receptor expression in breast cancer: the Multiethnic Cohort Study. Cancer Epidemiol. 2011;35:448-52. 